\title{
NHLBI Coronary Artery Dissection Type A
}

National Cancer Institute

\section{Source}

National Cancer Institute. NHLBI Coronary Artery Dissection Type A. NCI Thesaurus.

Code C119588.

Minor radiolucencies within the lumen during contrast injection with no persistence after dye clearance. (Adapted from: Coronary artery ang iog raphic changes after PTCA:

Manual of Operations NHLBI PTCA Registry 1985-6:9) 